\title{
Examining chronic pain through the lens of the socioeconomic gradient
}

\author{
Christophe Tanguay-Sabourin*
}

Integrated Program in Neuroscience, McGill University, Montreal, QC, Canada

${ }^{\star}$ Author for correspondence (christophe.tanguaysabourin@mail.mcgill.ca)

\begin{abstract}
:
Longstanding evidence reveals the existence of a gradient of health running along the socioeconomic spectrum. This is denoted by a graded association between health and levels of socioeconomic status, including factors such as gender, income, education, and occupational roles. This gradient is found across many chronic diseases including heart failure, arthritis, type 2 diabetes, ulcers, and certain cancers, all of which commonly possess debilitating pain diagnoses. Here, I examine chronic pain and its severity through the lens of this socioeconomic gradient across three perspectives along with their potential limitations. First, I discuss how this gradient represents risk factors for greater pain severity, disability, and comorbidity. Then, I explore potential underlying health determinants and how one's position on this spectrum may predetermine their chance of receiving optimal care for their pain. Finally, I end with the prospect of better clinical and biological understanding of chronic pain severity with the inclusion of this socioeconomic gradient.
\end{abstract}

\section{Introduction}

Pain is the leading utilization of health care resources and the most important source of disability among working adults in both Canada and the United States. In Canada, we estimate chronic pain to affect nearly $20 \%$ of the general population. The estimated cost of chronic pain to the Canadian society is $\$ 6$ billion/year in medical treatments and $\$ 37$ billion/year in loss of productivity [1]. Given its high prevalence and important societal cost, chronic pain has evolved from a common comorbidity to a prominent health concern of its own.

Unfortunately, the underlying etiology behind most chronic pain conditions, along with the mechanisms behind the transition from acute to chronic status, remains poorly understood [2]. The traditional injury model is not enough to explain pain and its chronicity. For patients suffering from low back pain, one of the most prevalent forms of chronic pain, only a small portion of patients $(<5 \%)$ report a traumatic event or fracture that could have contributed to their pain development [3]. This is not specific to back pain as most chronic pain types do not present a single specific etiology, but rather a mix.

This poor pain-injury correspondence has led to research targeting common mechanisms behind chronic pain conditions. Pain is a complex process including both a sensory-discriminative and an affective-motivational dimension, relying on detection mechanisms, reactive nociception, and reinforcement of avoidant behavior toward any pain or injury.
This has motivated further research toward a biopsychosocial model of pain, acknowledging the synergic contributions from biological, psychological, and social factors to the development or maintenance of chronic pain [4]. While research has increasingly started to combine biological and psychological measures to better understand chronic pain, social factors remain most often ignored. Researchers may control for social factors, but do not typically examine them despite the various health outcomes associated with socioeconomic status (SES).

\section{The socioeconomic gradient and its role in chronic pain}

The World Health Organization refers to SES as the social gradient of health, running from the top to the bottom of the socioeconomic spectrum. Denoted by a graded association between health and all levels of SES, this measure includes factors such as income, education, profession, and more. Additionally, demographic factors such as one's gender, age, and ethnicity also play a role in the socioeconomic gradient of health as they contribute to one's overall status and position in society.

Interestingly, this gradient is also found across many chronic diseases including heart failure, arthritis, type 2 diabetes, ulcers, and certain cancers, all of which present common debilitating pain diagnoses such as chest pain, osteo-
This is an Open Access article distributed under the terms of the Creative Commons Attribution License (CC BY 4.0)

License deed can be found at: http://creativecommons.org/licenses/ by $/ 4.0 /$ https://doi.org/10.29173/hsi300

Received 26 February 2020 Accepted 17 June 2020 
arthritis, diabetic neuropathy, burning stomach, and cancer pain. In parallel, extensive literature describes low SES as a strong potential risk factor for chronic pain. Population-wide studies reveal large socioeconomic disparities in pain across gender, education, and income. Pain levels are lower for men than women and decrease across increasing education and income quartiles [5]. A study examining the experience of pain showed that lower SES was associated with a higher prevalence of severe pain, including a greater number of painful body sites, greater pain intensity, and/or greater feelings of being disabled by pain. Even at the same intensity of pain and the same number of painful body sites, the study reported that participants at the lowest as compared to the highest SES were two to three times more likely to feel disabled by their pain [6]. This is in addition to the greater risk of developing multiple psychiatric comorbidities: generalized stress disorder, panic disorder, depression, and post-traumatic stress disorder along with their pain [7], also commonly observed in lower SES populations [8].

Importantly, demographics also contribute to the effects mentioned above within this socioeconomic gradient, with pain typically being more pronounced in women [5]. Pain-related disparities between men and women are both biological (sex) and socio-cultural (gender) in nature. Strong biological evidence indicates that males experience stronger opioid analgesia [9]. Yet, these differences are also the recipient of social influences. For instance, questionnaires assessing the gender role in pain expectation reveal that even after controlling for sex, self-identified gender still predicts pain tolerance [10]. It remains unclear, however, the extent to which we can dissociate the contribution of SES from gender, as women typically present lower SES compared to men. A very similar case can be made with ethnicity, where pain is found more prevalent and disabling among non-Caucasian ethnicities [5]. However, a study suggests that this race effect appears to be strongly mediated by socioeconomic disparities. While non-Caucasian populations are typically the first targets of this socioeconomic gradient, presenting lower income, education, and occupational role, non-Caucasian individuals living in neighborhoods of greater SES do not seem to present the same pain severity as those of lower SES [11]. However, little research has explained the potential biological role behind race in chronic pain, and it still requires further investigation.

\section{Health determinants of chronic pain and likelihood for optimal care}

Linking this socioeconomic gradient to chronic pain may not be enough to provide potential avenues towards understanding disease or treatment outcomes. One must understand the mechanisms driving this gradient that impact the subjective experience of pain. This is especially important given that these mechanisms may be overlapping across many chronic pain conditions.

One well-recognized determinant of health is health behaviors, including smoking, alcohol use, physical inactivity, poor sleep, and malnutrition, all of which have also been independently observed as predictors for greater prevalence or severity of chronic pain [12]. Another determinant of health, probably the most specific to lower SES and more impactful for chronic pain patients, is the access in health care and the related expenditure for successful treatment.

Populations on the lower end of the socioeconomic gradient have been found to be misrepresented in clinical trials [13], limiting the extent to which a given successful treatment is generalizable. The reported efficacy may thus be over-optimistic, as it doesn't account for the additional severity and comorbidities typically reported in these populations. Furthermore, these populations report increased prevalence of pain in the chronic ( $>6$ months), rather than acute $(<3$ months) phase. This suggests that SES may not play a role in the occurrence of pain, but instead in the transition from acute to chronic pain [14], potentially reflecting suboptimal treatment in these populations.

The poor understanding of chronic pain etiology has also led to multidisciplinary treatment approaches which are found to work best in reducing pain. In addition to opioid-derived medication, this includes an array of off-label medications (anticonvulsants, antidepressants, and others) paired with other forms of interventions (physical therapy, relaxation techniques, cognitive behavioral training, among many others). However, this approach must be done early in the acute phase ( $<3$ months) in order to be effective [15]. While individuals are in the acute-to-chronic transition phase (3-6 months), they are at greater risk of developing psychiatric comorbidities as a result of their pain and will typically become less responsive to traditional treatments in the future.

Unfortunately, this approach, now considered standard to chronic pain care, is costly in time and money. For individuals on the lower end of the socioeconomic gradient who often have less-accommodating work environments, their economic well-being suffers due to the cost of treatment and lost productivity. This is in addition to a variety of complementary and alternative medicine (CAM) treatment forms typically recommended during the multidisciplinary treatment approach not accessible due to their extraneous cost. CAM therapy users are typically younger, more highly educated, and have a higher income [16]. Patients presenting lower SES are also less likely to be prescribed traditional opioid treatments due to being at higher risk for substance abuse disorders, despite it being the most effective treatment for pain management in the acute phase.

One major limitation in the interpretation of the determinants of health towards this socioeconomic gradient is directionality. On one hand, socioeconomic factors may predispose individuals to turn to different coping mechanisms (e.g. smoking, alcohol use, malnutrition) for their pain dependent upon their affordability. These coping techniques, although potentially beneficial to cope with pain in the short-term, can lead to further morbidity or mortality in the long-term. This is in addition to other health behaviors that 
may reflect reaction mechanisms to intolerable pain (e.g. poor sleep and physical inactivity). On the other hand, one may suggest that poor health behaviors may predispose a given individual to experience greater pain, as each of these behaviors is essential to optimal health and pain tolerance. The relationship between health behaviors and chronic pain may be bidirectional, and therefore self-reinforcing in the maintenance or worsening of chronic pain.

Similarly, the directionality also applies to access to health care. Patients who develop chronic pain following a traumatic event may be more likely to transit toward a lower socioeconomic status as a result of their pain. The exorbitant health care cost along with their inability to sustain the type of work they normally engage in may lead to unemployment, decreased income, and an inability to pursue further education. A careful interpretation is therefore needed before declaring specific directionality of these health determinants with chronic pain.

\section{Understanding chronic pain etiology \& biology through SES}

Investigating the socioeconomic gradient and its health implications is an important, yet often overlooked component in our understanding of pain and its chronicity. Human neurobiological research has so far presented very few models that reliably reflect pain severity in patients, mostly limited to modeling chronic pain as a dichotomous outcome in patients (i.e. present or absent). These measures, including brain imaging, biological sampling, and genetics among many more, typically present poor correlates of pain severity (i.e. as a continuous variable) in comparison to psychosocial variables [17].

In this case, the lack of ecological consideration may be hindering biological understanding, ignoring the various health determinants contributing to the maintenance, worsening, or potential resilience of chronic pain. This idea for ecological or holistic consideration, however, is not new nor specific to chronic pain. There has been a growing trend toward moving away from small, carefully selected, and homogeneous cohorts toward larger-scale cohorts presenting richer and more diverse phenotyping, potentially more representative. This has also been accompanied by an interest toward normative modeling of populations, acknowledging disease heterogeneity and potential transdiagnostic similarities in treating these conditions as a spectrum rather than discrete categories. This approach seems ideal for the study of chronic pain.

Fortunately, the prospect of integrating this socioeconomic gradient within our biological understanding of chronic pain may be currently possible. A population-wide study that has enrolled nearly one million adults in the United Kingdom Biobank (i.e. the UK Biobank) will investigate the contribution of biological [brain imaging, biological sampling, and genetic], environmental (demographics and socioeconomic), and various health outcomes (pain, sleep, nutrition, and more) in the development of diseases. This rep- resents by far the largest sample ever studied of chronic pain patients and an exciting prospect for understanding chronic pain across diseases along with their interactions with SES. Furthermore, the researchers intend to contact participants five to ten years following the initial assessment, providing an opportunity for the study of the combination of these socioeconomic and biological factors in the acute-to-chronic pain transition.

While socioeconomic factors may contribute to providing a better understanding of pain severity, it may lead to further consideration for factors contributing uniquely to each of these chronic pain diseases. Although the common sociodemographic mechanisms behind chronic pain are conceptually similar, distinct mechanisms of health are also occurring. For instance, chronic back pain is most prevalent in blue-collar workers while painful diabetic neuropathy is more prevalent in populations suffering from malnutrition and obesity. The effect of gender may also differ depending on the disease. Chest pain is typically more common in men, while widespread pain is more common in women. This also applies to biological mechanisms, where some chronic pain conditions present well-established markers. This is the case for a rheumatoid factors test that can help track the number of autoantibodies in the blood and help pinpoint a diagnosis for rheumatoid arthritis. Although it may correlate poorly with pain, it may be essential in tracking the disease progression.

\section{Conclusion}

Here, we denote that chronic pain severity appears to be intimately related to one's position on the socioeconomic gradient. Given that many key determinants of pain are the social and economic conditions in which people work and live, research must be directed at the root of those socioeconomic factors shaping pain and suffering. This is true for the whole spectrum, including why individuals on the higher end of the spectrum present less pain severity. What factors do patients with higher SES present that alleviate their pain, beyond these health determinants? Could they be targeted specifically?

On one hand, there is great potential for furthering our understanding of pain severity and the common biology among chronic pain conditions. On the other hand, this gradient of health also entails a clear need for the development of a comprehensive healthcare plan based on a patient's socioeconomic profile and may force us potentially redefine what we think of as minimal health coverage. This may be a crucial step toward more accessible pain management which will become increasingly important in our aging society. 


\section{References}

1. Lynch ME. The Need for a Canadian Pain Strategy. Pain Res Manag [Internet]. 2011;16(2):77-80. Available from: http://www.hindawi. com/journals/prm/2011/654651/

2. Treede R-D, Rief W, Barke A, Aziz Q, Bennett MI, Benoliel R, et al. A classification of chronic pain for ICD-11. Pain [Internet]. 2015 Mar;1. Available from: http://journals.lww.com/00006396900000000-99874

3. Chen KY, Shaparin N, Gritsenko K. Low Back Pain. In: Pain Medicine [Internet]. Cham: Springer International Publishing; 2017. p. 461-3. Available from: http://link.springer.com/10.1007/978-3-31943133-8_121

4. Lumley MA, Cohen JL, Borszcz GS, Cano A, Radcliffe AM, Porter LS, et al. Pain and emotion: a biopsychosocial review of recent research. J Clin Psychol [Internet]. 2011 Sep;67(9):942-68. Available from: http://doi.wiley.com/10.1002/jclp.20816

5. Grol-Prokopczyk H. Sociodemographic disparities in chronic pain, based on 12-year longitudinal data. Pain [Internet]. 2017 Feb;158(2):313-22. Available from: http://journals.lww. com/10.1097/j.pain.0000000000000762

6. Dorner TE, Muckenhuber J, Stronegger WJ, Ràsky É, Gustorff B, Freidl W. The impact of socio-economic status on pain and the perception of disability due to pain. Eur J Pain [Internet]. 2011 Jan;15(1):103-9. Available from: http://doi.wiley.com/10.1016/j. ejpain.2010.05.013

7. McWilliams LA, Cox BJ, Enns MW. Mood and anxiety disorders associated with chronic pain: an examination in a nationally representative sample. Pain [Internet]. 2003 Nov;106(1):127-33. Available from: http://content. wkhealth.com/linkback/openurl?sid=WKPTLP:landingpage\& an $=00006396-200311000-00017$

8. Miech RA, Caspi A, Moffitt TE, Wright BRE, Silva PA. Low Socioeconomic Status and Mental Disorders: A Longitudinal Study of Selection and Causation during Young Adulthood. Am J Sociol [Internet]. 1999 Jan;104(4):1096-131. Available from: https://www. journals.uchicago.edu/doi/10.1086/210137

9. Fillingim RB, Ness TJ. Sex-related hormonal influences on pain and analgesic responses. Neurosci Biobehav Rev [Internet]. 2000 Jun;24(4):485-501. Available from: https://linkinghub.elsevier.com/ retrieve/pii/S0149763400000178

10. Wise EA, Price DD, Myers CD, Heft MW, Robinson ME. Gender role expectations of pain: relationship to experimental pain perception. Pain [Internet]. 2002 Apr;96(3):335-42. Available from: http:// content.wkhealth.com/linkback/openurl?sid=WKPTLP:landingpage \&an=00006396-200204000-00015

11. Fuentes M, Hart-Johnson T, Green CR. The association among neighborhood socioeconomic status, race and chronic pain in black and white older adults. J Natl Med Assoc [Internet]. 2007 Oct;99(10):1160-9. Available from: http://www.ncbi.nlm.nih.gov/ pubmed/17987920

12. van Hecke O, Torrance N, Smith BH. Chronic pain epidemiology - where do lifestyle factors fit in? Br J Pain [Internet]. 2013 Nov 19;7(4):209-17. Available from: http://journals.sagepub.com/ doi/10.1177/2049463713493264

13. Sharrocks K, Spicer J, Camidge DR, Papa S. The impact of socioeconomic status on access to cancer clinical trials. Br J Cancer [Internet]. 2014 Oct 5;111(9):1684-7. Available from: http://www.nature. com/articles/bjc2014108

14. Saastamoinen P, Leino-Arjas P, Laaksonen M, Lahelma E. Socio-economic differences in the prevalence of acute, chronic and disabling chronic pain among ageing employees. Pain [Internet]. 2005 Apr;114(3):364-71. Available from: http://content. wkhealth.com/linkback/openurl?sid=WKPTLP:landingpage\& an $=00006396-200504000-00009$

15. Kamper SJ, Apeldoorn AT, Chiarotto A, Smeets RJEM, Ostelo RWJG, Guzman J, et al. Multidisciplinary biopsychosocial rehabilitation for chronic low back pain. Cochrane Database Syst Rev [Internet]. 2014 Sep 2; Available from: http://doi.wiley. com/10.1002/14651858.CD000963.pub3

16. Braverman DL, Ericksen JJ, Shah R V., Franklin DJ. 3. New frontiers in pain management: Complementary techniques. Arch Phys Med Rehabil [Internet]. 2003 Mar;84:S45-9. Available from: https://linkinghub.elsevier.com/retrieve/pii/S0003999302048670
17. Meints SM, Edwards RR. Evaluating psychosocial contributions to chronic pain outcomes. Prog Neuro-Psychopharmacology Biol Psychiatry [Internet]. 2018 Dec;87:168-82. Available from: https:// linkinghub.elsevier.com/retrieve/pii/S0278584617309855

(C) The Author(s) 